\title{
Validación de la versión en español de Motivation and Engagement Scale-Music para la evaluación de la motivación en estudiantes de música
}

\author{
Mtra. Zita Guadalupe Contreras Hernández \\ zitag.contreras@gmail.com \\ Dr. Daniel González, Lomelí \\ Universidad de Sonora \\ Posgrado Integral en Ciencias Sociales
}

Resumen. El objetivo de la presente investigación fue probar la validez y confiabilidad de una versión adaptada al español de la escala Motivation and Engagement Scale-Music (MES-M, Martin, 2008) para evaluar la motivación y el compromiso que perciben los estudiantes de música sobre su propia actividad musical. La muestra fue conformada por 96 estudiantes de música que cursan en la Licenciatura en Música y los Talleres Libres de la Universidad de Sonora. Los resultados permitieron confirmar las propiedades psicométricas del cuestionario, en donde las escalas presentaron buena consistencia interna, con valores de Cronbach arriba de .60. La validez de constructo y la estructura teórica fueron comprobadas mediante un análisis factorial confirmatorio y el modelo resultante estuvo conformado por cinco dimensiones y dos factores de segundo orden, mostraron un buen ajuste con los datos. Estos resultados apoyan el uso de la escala para evaluar la motivación y el compromiso que tienen los estudiantes hacia su propia actividad musical. Se concluye que la motivación y el compromiso en la actividad musical pueden ser teóricamente explicados a través de diversos dominios de desempeño.

Palabras clave: Motivación, Compromiso, Estudiantes de música, Análisis factorial.

\section{Introducción}

La motivación y el compromiso desempeñan un papel importante en el interés y disfrute de actividades que las personas realizan. El rendimiento que tienen los estudiantes de música puede verse influenciado por la evaluación que hacen ellos mismos con respecto a su ejecución musical (Jones, 2009).

Según Martín (2007) la motivación se refiere a la energía y dirección de uno mismo para aprender y desarrollar todo su potencial; el compromiso se refiere a conductas guiadas bajo esta motivación. En el campo de la investigación científica, la temática de la motivación se encuentra fragmentada, es por esto que Martin (2007) desarrolló una teoría para integrar las distintas aproximaciones acerca de la investigación sobre la motivación. Para entender la motivación y el compromiso, el autor se basó en la integración de varias contribuciones teóricas como la teoría de la necesidad de logro y teorías sobre motivación y autoestima (Covington y Omelich, 1991), teoría de 
la autoeficacia (Bandura, 1986, 1997), teoría de la expectativa y valoración (Eccles, 1983), teoría de la atribución (Weiner, 1985), teoría sobre el control (Conne11, 1985), teoría de la auto-determinación (Ryan y Deci, 2000), y teoría de la motivación y orientación (Duda y Nicholls, 1992). En conjunto, estas teorías explican por qué los individuos hacen lo que hacen, cómo lo hacen, su confianza en ser capaz de hacer lo que hacen, y su habilidad de superar obstáculos y retos. Bajo esta perspectiva, el autor le da dirección a las dimensiones específicas de la motivación y el compromiso en un modelo llamado la "Rueda de la Motivación" (2007) la cual se compone de cuatro factores de segundo orden: cognición adaptativa e inadaptada y comportamiento adaptativo e inadaptado; que integran 11 factores de primer orden: autoeficacia, valoración, orientación al dominio, planeación, administración de tarea, persistencia, evitar el fracaso, control incierto, ansiedad, auto-discapacidad y descompromiso.

De acuerdo a Martin (2007) la cognición adaptativa es representada por:

- Autoeficacia, se refiere a la confianza en las capacidades de uno mismo para realizar de manera eficaz tareas y actividades.

- Valoración, referida a las creencias sobre la importancia, utilidad y relevancia de las tareas y actividades que uno realiza.

- Orientación al dominio, definida como una orientación dirigida al desarrollo, entendimiento y conocimientos de una actividad realizada.

El comportamiento adaptativo es representado por:

- Planeación, se refiere al nivel de planeación que se lleva a cabo como preparación para realizar alguna actividad.
- Administración de tareas, se refiere a la habilidad para utilizar el tiempo y el espacio físico de manera efectiva de la actividad que se desee realizar.

- Persistencia, se refiere a la aplicación sostenida del esfuerzo en medio de las dificultades o desafíos.

La cognición inadaptada es representado por:

- Evitar el fracaso, motivación que está dirigida por un deseo de evitar hacer mal las cosas y decepcionar a los otros.

- Control incierto, se refiere a la sensación de ser incapaz de controlar los resultados futuros con respecto a las tareas y actividades de uno.

- Ansiedad, es un sentimiento de nerviosismo y preocupación con respecto a la tarea que se requiere realizar.

El comportamiento inadaptado se encuentra representado por:

- Auto-sabotaje, se refiere a sabotear las posibilidades de éxito, como la procrastinación, de manera que se generan excusas en caso de un mal rendimiento en la tarea.

- "Descompromiso", se refiere a la sensación de darse por vencido.

El modelo multidimensional de la motivación y el compromiso propuesto por el autor fue aplicado primeramente en el ámbito académico (Martin, 2007) y después se confirmó la bondad de ajuste del modelo en otros ámbitos como la música y el deporte, contextos que carecen de una fundamentación teórica sobre la motivación y el compromiso de participar en dichas actividades (Martin, 2008).

\section{Método}

El objetivo de la presente investigación fue probar la validez y confiabilidad de una versión adaptada al español de 
la escala Motivation and Engagement Scale-Music (MES-M, Martin, 2008) para evaluar la motivación y el compromiso que perciben los estudiantes de música sobre su propia actividad musical. Se trabajó con un enfoque cuantitativo, de tipo no experimental, transversal, con un diseño descriptivo. En una primera fase se realizó la traducción y adaptación al español del cuestionario por medio de un experto bilingüe. El estudio se presentó a los participantes como una investigación sobre la relación entre la música y el bienestar. Tras informar a los participantes de que toda la información recogida en la investigación sería confidencial y anónima, se les entregó el cuestionario.

\section{Participantes}

El estudio se realizó durante las horas de clase del semestre 2016-1 en las aulas de la escuela de música de la Universidad de Sonora. Participaron en el estudio un total de 96 estudiantes de música, de los cuales 56 estaban inscritos en la Licenciatura en Música y 40 estudiantes inscritos en los Talleres Libres de Música de la Universidad de Sonora. De la muestra total 36 participantes fueron mujeres $(34.7 \%)$ y 60 hombres $(60.2 \%)$, cursando sus estudios desde los Talleres Libres, hasta séptimo semestre en nivel licenciatura. La edad de los participantes se distribuye en un rango entre 13 y 73 años, con una edad media de 25.49.

\section{Instrumento}

Para medir la motivación y el compromiso con la actividad musical, se utilizó la traducción al español de la escala Motivation and engagement-Music, la cual se compone de 11 factores de primer orden (autoeficacia, valoración, orientación al dominio, planeación, administración de tareas, persistencia, ansiedad, evitar el fracaso, control incierto, auto-discapacidad y descompromiso); y 4 factores de segundo orden (cognición y comporta- miento adaptativo y cognición y comportamiento inadaptado). Dicha escala está compuesta de un total de 44 reactivos, 4 ítems para cada subdimensión en una escala de 7 puntos que va desde " $1 "=$ Muy en desacuerdo a "7" = muy de acuerdo.

Ejemplo de reactivos:

a) Autoeficacia: si practico mucho creo que puedo tocar bien mi música.

b) Orientación al dominio: me siento muy satisfecho conmigo mismo cuando aprendo cosas nuevas de música.

c) Planeación: trato de planear las cosas antes de empezar algo en mi música.

d) Administración de tareas: cuando me preparo para presentaciones musicales, por lo general lo hago en un lugar en donde pueda concentrarme.

e) Persistencia: si no puedo entender algo en mi música la primera vez, sigo dándole vueltas hasta entenderlo.

f) Ansiedad: me preocupa tocar mal mi música.

g) Evitar el fracaso: con frecuencia, la razón principal por la que trato de tocar mi música es porque no quiero decepcionar a personas cercanas a mí.

h) Control incierto: cuando no toco bien mi música, con frecuencia no estoy seguro como evitar que esto me suceda de nuevo.

i) Auto-sabotaje: a veces hago otras cosas en vez de practicar música para así tener un pretexto si no toco bien.

j) "Descompromiso": ya no me importa mi música.

\section{Análisis de datos}

Los datos fueron capturados y analizados utilizando el paquete estadístico SPSS versión 21 . Se obtuvieron las medidas de tendencia central y desviaciones estándar para los datos sociodemográficos, y para las variables y dimensiones de cada escala. Se consideraron valores mayores a .60 en las alfas de Cronbach para la compro- 
bación de confiabilidad. El análisis factorial confirmatorio se realizó por medio de un análisis de ecuaciones estructurales (Bentler, 2006). Se utilizó el programa estadístico de ecuaciones estructurales (EQS) para medir la bondad de ajuste de los modelos factoriales propuestos por cada escala y para conocer la distribución de los factores en relación con cada uno de los indicadores.

\section{Resultados}

La tabla 1 presenta los estadísticos descriptivos los cuales muestran las medias y la consistencia interna de las dimensiones y reactivos de la escala de Motivación y compromiso con la actividad musical, la cual se conformó con un total de 44 reactivos, compuesta por 11 subescalas, en donde se obtuvo una media total de 4.61 , con un índice de consistencia interna de .83. La dimensión de Autoeficacia fue conformada por 4 reactivos, donde se obtuvo una media de 6.43 y un alfa de .67; la dimensión de Valoración fue conformada por 4 reactivos, se obtuvo una media de 6.35 y un alfa de .67; para la dimensión de Orientación al Dominio se obtuvo una media de 6.56, un alfa de .71 y estuvo conformada por 4 reactivos; la dimensión de Planeación obtuvo una media de 5.24, un alfa de .70 y fue conformada por 4 reactivos; la dimensión de Administración de Tareas fue conformada por 4 reactivos, con una media de 5.78 y un índice de consistencia interna de .88; la escala de Persistencia fue conformada por 4 reactivos, con una media 6.00 de y una consistencia interna de .85; la escala de Evitar el fracaso estuvo conformada por 4 reactivos y obtuvo una media de 2.88 con un índice de consistencia interna de .87; para la dimensión de Control incierto se obtuvo una media de 3.17 , un alfa de .87 y estuvo conformada por 4 reactivos; la dimensión de Ansiedad obtuvo una media de 4.58 , un alfa de .71 y fue conformada por 4 reactivos; la dimensión de Auto-sabotaje fue conformada por 4 reactivos con una media de 2.23 y un alfa de .87; por último, la escala de descompromiso musical estuvo compuesta por 4 reactivo que obtuvieron una media de $1.96 \mathrm{y}$ un alfa de .67.

Tabla 1

\begin{tabular}{lcccccc}
$\begin{array}{l}\text { Análisis de consistencia interna } \\
\text { Compromiso-Música }\end{array}$ & estadisticos & descriptivos & de & la & escala & Motivación $y$ \\
\multicolumn{1}{c}{ Escalas/Reactivos } & $N$ & $M I N$ & $M A X$ & $M$ & $D E$ & $\alpha$ \\
& & & & & & \\
\hline 1.Cognición Adaptativa & & & & $\mathbf{6 . 4 1}$ & & .84 \\
Autoeficacia & 96 & 4.50 & 7.00 & 6.43 & .668 & .67 \\
Valoración & 96 & 3.75 & 7.00 & 6.35 & .689 & .67 \\
Orientación al dominio & 96 & 4.25 & 7.00 & 6.56 & .638 & .71 \\
2.Comportamiento Adaptativo & & & & 5.68 & & .90 \\
Planeación & 96 & 1.50 & 7.00 & 5.24 & 1.01 & .70 \\
Administración de tarea & 96 & 1.50 & 7.00 & 5.78 & 1.108 & .88 \\
Persistencia & 96 & 2.00 & 7.00 & 6.00 & .951 & .85 \\
3.Cognición inadaptada & & & & 3.40 & & .84 \\
Evitar el fracaso & 96 & 1.00 & 7.00 & 2.38 & 1.367 & .87 \\
Control incierto & 96 & 1.00 & 7.00 & 3.17 & 1.515 & .87 \\
Ansiedad & 95 & 1.00 & 7.00 & 4.58 & 1.264 & .71 \\
4.Comportamiento inadaptado & & & & $\mathbf{2 . 0 8}$ & .87 \\
Auto-sabotaje & 96 & 1.00 & 7.00 & 2.23 & 1.229 & .87 \\
Descompromiso & 96 & 1.00 & 6.25 & 1.96 & .979 & .67
\end{tabular}

Figura 1. Modelo factorial confirmatorio de motivación y desmotivación musical. X2=111.811, (97gl), $p=.144$, BBNFI: .820, BBNNFI: .963, CF1: .970, RMSEA: .04, Alpha $=.69$ 
En la figura 1 se muestran los resultados del análisis factorial confirmatorio para la escala Motivación y Compromiso con la actividad musical. Para realizar éste análisis se utilizó el programa de ecuaciones estructurales (EQS). Durante la corrida del programa no se encontraron problemas durante la optimización; con esto se puede considerar que los resultados son confiables. La X2 resultante fue de 111.81 (97gl), asociada a una $\mathrm{p}=.144)$; el NFI fue de .82 , el NNFI fue de .96 y el CFI fue de .97. Esto indica que el modelo resultante que presenta mejor bondad de ajuste está conformado por cinco factores de primer orden (autoeficacia, planeación, ansiedad, auto-sabotaje y descompromiso) y dos factores de segundo orden (motivación y desmotivación musical), pues los indicadores de bondad de ajuste son mayores a .90 (NNFI y CFI). Se formaron coherentemente los 5 factores de primer orden, ya que los pesos factoriales de cada uno fueron altos y significativos. Con esto se puede considerar que el modelo factorial muestra validez convergente, pues los indicadores de cada factor convergen entre sí dado el valor alto y significativo de los pesos factoriales.

\section{Conclusiones}

A modo de resumen, la conclusión principal que podemos extraer de este trabajo de investigación es que la motivación y el compromiso pueden ser teóricamente explicados a través de diversos dominios de desempeño. Tal como lo propone el autor Martin (2008), constructos como autoeficacia, planeación, ansiedad y descompromiso son relevantes en cualquier situación en donde los individuos requie- ran desempeñar una actividad y ser evaluados de alguna manera.

De acuerdo a los resultados obtenidos, los estudiantes de música de la licenciatura y talleres libres de la Universidad de Sonora, presentan puntuaciones altas en las variables relacionadas con la motivación y el compromiso (autoeficacia $=6.43$, planeación $=5.24)$ y reportan puntuaciones bajas en las variables relacionadas con la desmotivación musical (auto-sabotaje= 2.23, descompromiso=1.96). Sin embargo, reportaron puntuaciones relativamente altas con respecto a la variable ansie$\mathrm{dad}=4.58$.

La escala utilizada en el presente estudio es un instrumento comprensible para los estudiantes de música y es equivalente a la versión original. Muestra buenas propiedades psicométricas, consistencia interna y validez de constructo. Los resultados muestran puntuaciones similares, reforzando la validez del cuestionario en el ámbito de educación musical a nivel superior.

Disponer de un instrumento específicamente diseñado para medir motivación y compromiso en estudiantes de música, adaptado de la versión original y con buenas propiedades como instrumento de medida, permitirá llevar a cabo estudios que contribuyan a mejorar la calidad de la educación musical en nuestro país. Se hace evidente que la práctica educativa necesita este tipo de trabajos con conclusiones que sirvan de base para llevar a cabo programas de intervención, mejorando la calidad de la enseñanza a través de un currículum y docencia más eficiente.

\section{Bibliografía}

Bandura, A. (1996). Social foundations of thought and action: A social cognitive theory. New Jersey: Prentice Hall.

Bandura, A. (1997). Self-efficacy: The exercise of control. New York: Freeman \& Co. 
Bentler, P. M. (2006). EQS Structural Equations Program, Manual Multivariate Software.

Connel, J.P. (1985). A new multidimensional measure of children's perceptions of control. Child Development, 56, 1018-1041.

Duda, J.L. (1992). Motivation in sport settings: A goal perspective approach. In G.C. Roberts (Ed.). Motivation in sport and exercise. Champaign, IL: Human Kinetics Books.

Jones, B . (2009). Motivating Students to Engage in Learning: The MUSIC Model od Academic Motivation. International Journal of Teaching and Learning in Higher Education. 21(2), 272-285.

Kemp, A. E. (1997). Individual differences in musical behaviour. In D. J. Hargreaves \& A. C. North (Eds.), The social psychology of music (pp. 25-45). Oxford: Oxford University Press

Martin, A.J. (2007). Examining a multidimensional model of student motivation and engagement using a construct validation approach. British Journal of Educational Psychology, 77, 413-440.

Martin, A.J. (2007). Examining a multidimensional model of student motivation and engagement using a construct validation approach. British Journal of Educational Psychology, 77, 413-440.

Martin, A.J. (2008). Motivation and Engagement in Music and Sport: Testing a Multidimensional Framework in Diverse Performance Settings. Journal of Personality, 76(1), 135-170.

Martin, A.J. (2008). Motivation and Engagement in Music and Sport: Testing a Multidimensional Framework in Diverse Performance Settings. Journal of Personality, 76(1), 135-170.

Ryan, R.M., \& Deci, E.L. (2000). Self-determination theory and the facilitation of intrinsic motivation, social development, and well-being. American Psychologist, $55,68-78$.

Weiner, B. (1985). An attributional theory of achievement motivation and emotion. Psychological Review, 92, 548-573.

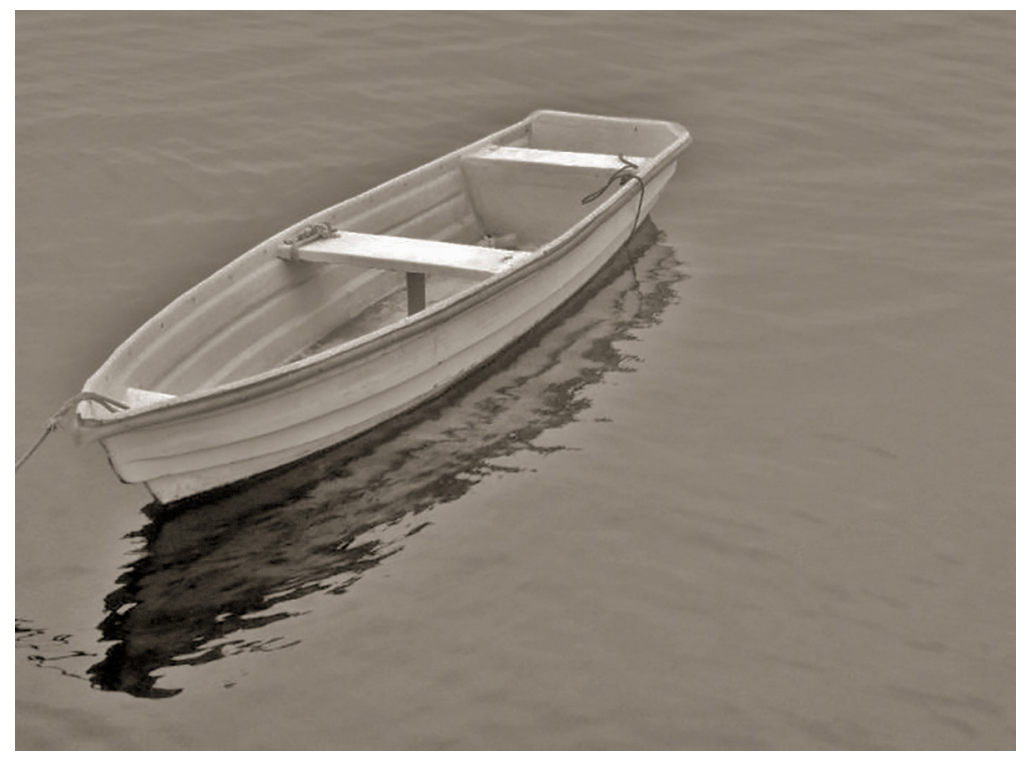

